\title{
Ultra High Vacuum Chemical Vapor Deposition Techniques for Economic Growth of Silicon Nanowires
}

\author{
Rashad Filfilan $^{1}$, Maha Khayyat ${ }^{2,}$ * \\ ${ }^{1}$ Midra Tower, Saudi Aramco, Dhahran, Saudi Arabia \\ ${ }^{2}$ Materials Science Research Institute, King Abdullaziz City for Science and Technology, Riyadh, Saudi Arabia
}

Email address:

mkhayyat@kacst.edu.sa (M. Khayyat)

${ }^{*}$ Corresponding author

To cite this article:

Rashad Filfilan, Maha Khayyat. Ultra High Vacuum Chemical Vapor Deposition Techniques for Economic Growth of Silicon Nanowires. American Journal of Nanosciences. Vol. 7, No. 2, 2021, pp. 42-48. doi: 10.11648/j.ajn.20210702.12

Received: July 23, 2021; Accepted: August 6, 2021; Published: August 11, 2021

\begin{abstract}
Ultra High Vacuum Chemical Vapor Deposition (UHVCVD) reactor has been used to grow silicon nanowires via innovative economical approaches using chemical active materials as catalysts such as aluminum. Scanning Electron Microscopy has been used to study the success of the growth for further investigations and advanced applications such as solar cells. Solar manufacturers are looking for approaches to improve yield and cell efficiency and lower manufacturing costs overall. One of the main goals of this project is to tear down the various parameters involved in the current photovoltaic panels and improve it in one or more directions (properties, performance, costs). The current study is addressing the solar market with special concern of the efficiency goal. The mechanical flexibility of plastic materials is of high demands for all photovoltaic applications onto curved surfaces for architectural integration. Polycarbonate AND/OR poly methyl methacrylate encapsulation of photovoltaic modules and usage to fabricate advanced silicon nanowires solar cells can be emerging technologies delivering excellent performance and durability at a competitive cost. Although solar panels with glass protective facing still account for the majority of the installations of photovoltaic modules, however, it is expected that the adaptation of these new technologies will rapidly gain market share. The growth of silicon nanowires using chemical vapor deposition to fabricate advanced solar cells can be done via two innovative approaches. Alternative techniques for lithographic formation of the mask can provide advantages for low-cost processing, especially where a simple repeating pattern is required.
\end{abstract}

Keywords: Silicon, Nanowires, Ultra High Vacuum Chemical Vapour Deposition, Photovoltaic,

Scanning Electron Microscopy

\section{Introduction}

Polycarbonate (PC) AND/OR poly methyl methacrylate (PMMA) commonly used in eye protection, as well as in other projectile-resistant viewing and lighting applications that would normally indicate the use of glass, but require much higher impact-resistance. Moreover, there are applications, such as solar modules, which require higher impact-resistance along with good mechanical properties such as high tensile, shear strength, high modulus of elasticity, and easy to fabricate \& machine. These properties, plus good low frequency and high voltage insulating characteristics, make PC and PMMA carrier materials for electrical and electronic components [1-3]. It has been established that the properties of the commercial $\mathrm{PC}$ can be altered by various techniques [2], in regards to change its wettability, adhesion, and surface mechanical properties. At extreme weather conditions of high temperatures, sand, strong wind, rain, and sometimes hail PMMA can be considered as a good candidate replacing PC, with high clarity over longer periods of time. PMMA is relatively hard, lightweight material, however, its impact strength is significantly lower than PC. It has a density of $1.17-1.20 \mathrm{~g} / \mathrm{cm}^{3}$, which is less than half that of glass. [1] PMMA light transmittance is approximately $92 \%$ of visible light under standard conditions at $3 \mathrm{~mm}$ 
material thickness, and light reflection from its planner surface is in the range of less than $5 \%$ due to its refractive index 1.4905 at wavelength of $589.3 \mathrm{~nm} \mathrm{[3].} \mathrm{Most}$ importantly, PMMA has low water vapor absorption capacity makes it very suitable for electrical engineering purposes [4]. These properties of PMMA make it suitable for products intended for long open-air operation, such as PV cells and modules. Moreover, PMMA can be exist in different forms including sheets and micro- down to nanospheres. Where PC or PMMA planar sheets can be used to encapsulate PV panels, and the microspheres represent an innovative approach in solar cells advanced fabrication technique, as the current study is presenting.

A promising recently emerged field for future low cost, decent efficiency solar cell devices is the use of vaporliquid-solid (VLS) grown silicon nanowires ( $\mathrm{Si}-\mathrm{NWs}$ ). Bottom-up approach of Si-NWs growth via VLS mechanism has a key advantage for device applications, since it is possible to template the position of the NWs by controlling the placement of the initial metal seed or catalyst particle. This templating then allows integration of NWs with other parts of the structure, as required for many of these applications [5-8]. It has been proposed by Khayyat et al. 2013 [6] that the technique of microsphere lithography to produce regular hexagonal arrays of Alseeded nanowires, and the fabrication process continues to fabricate solar cells [7 \& 8]. It has been reported that PMMA nanospheres were used to increase the surface area of functional surfaces and other desired properties [1012]. One of the key goals of the current article is to create new application markets for acrylic such as applications for PC AND/OR PMMA in solar engineering and other alternate energy technologies.

The relative mechanical flexibility of thin plastic materials is of high demands for various technological applications including PV cells and modules. This allows the integration of this curved light weight module surfaces with architectural sites. Polycarbonate AND/OR poly methyl methacrylate encapsulation of photovoltaic modules and usage to fabricate advanced silicon nanowires solar cells can be emerging technologies delivering excellent performance and durability at a competitive cost. Although solar panels with glass protective facing still account for the majority of the installations of photovoltaic modules, however, it is expected that the adaptation of these new technologies will rapidly gain market share. The growth of silicon nanowires using Ultra High Vacuum Chemical Vapor Deposition UHVCVD to fabricate advanced solar cells can be done via two innovative approaches. Alternative techniques for the control growth of Si-NWs using the conventional photo or e-beam lithography in addition to the innovative micro- or nano-spheres can provide advantages for low-cost processing, especially where a regular repeating pattern is required [9-12].

\section{Experimental $[5,6]$}

$\mathrm{Si}-\mathrm{NWs}$ in the current investigation were grown in a home-built (UHVCVD) tool operated under extremely LowPressure conditions (LPCVD). A thermal Al evaporator and a monitor of quartz crystal are installed in the load lock chamber. The load lock chamber has a base pressure of $1 \times$ $10^{-7}$ Torr, and the UHVCVD growth chamber has a base pressure of $4 \times 10^{-9}$ Torr at room temperature and $2 \times 10^{-8}$ Torr at $600^{\circ} \mathrm{C}$. Si-NWs, as previously observed [5], grow preferentially perpendicularly in the (111) direction so $\mathrm{Si}(111)$ substrates were primarily used. The detailed growth conditions along with the pre-growth steps were described in previous work [5-8].

It has been shown, according to Khayyat et al [5], that as the thickness of Al catalyst layer is increased (from 2 $\mathrm{nm}$ to $5 \mathrm{~nm}$ ) the fidelity of the growth increases, then after reaching $5 \mathrm{~nm}$ Al thickness then the growth fidelity decreases quite significantly. Based on these measurements, it has been decided to choose $5 \mathrm{~nm}$ of $\mathrm{Al}$ thickness. When the desired Al thickness, $5 \mathrm{~nm}$, had been deposited, the substrates were moved out of the Al flux and the Al source was cooled. The samples were then transferred into the hot growth chamber for a pre-growth anneal at $600^{\circ} \mathrm{C}$, which is above the $\mathrm{Al} / \mathrm{Si}$ bulk eutectic temperature of $577^{\circ} \mathrm{C}$. This anneal was $20 \mathrm{~min}$ long under full pumping and was intended to allow the Al film to agglomerate into small islands on the surface and form liquid droplets. The furnace was then cooled to the desired growth temperature (usually $520^{\circ} \mathrm{C}$ ) and pure silane $\left(\mathrm{SiH}_{4}\right)$ was introduced at a fixed flow, $20 \mathrm{sccm}$, using a mass flow controller (MFC). Examining the Si-NWs morphology were examined with an environmental scanning electron microscope (SEM) FEI Co., Eindhoven, The Netherlands, model XL 30.

\section{Results and Discussion}

The vapor-liquid-solid (VLS) process (see figure 1) is well suited to producing structures with precisely controlled dimensions. NW growth occurs at the locations of the seed particles; the diameter of the NW is proportional to the seed volume; and compositional changes can be achieved by modulating the source gas flow [5-7].

Aligned, epitaxial growth can be achieved if a single crystal substrate is used, while random growth directions are seen on amorphous substrates [8]. Several techniques for controlling the placement, density, and diameter of noble metal seeds have been reviewed by Fan et al [9]. Electronbeam lithography (EBL) based patterning techniques are widely used in research but are less well suited for manufacturing because of their cost and low throughput. There are several Si-NWs growth techniques have been undertaken in this study, as follows; 
(a) Nucleation of the metallic nanoparticles
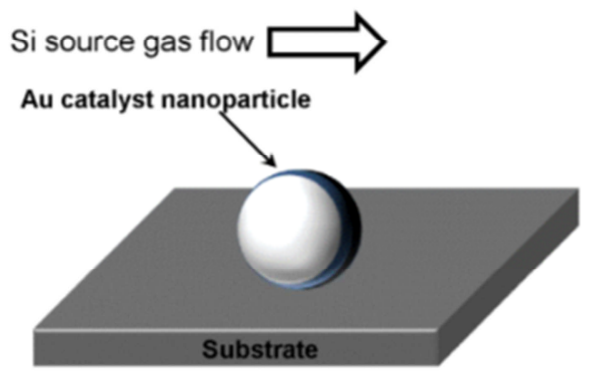

(c) Diffusion of Si vapors through the eutectic catalyst

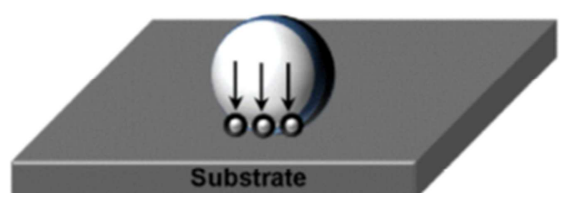

(b) Barrier gas decomposition into silicon vapor

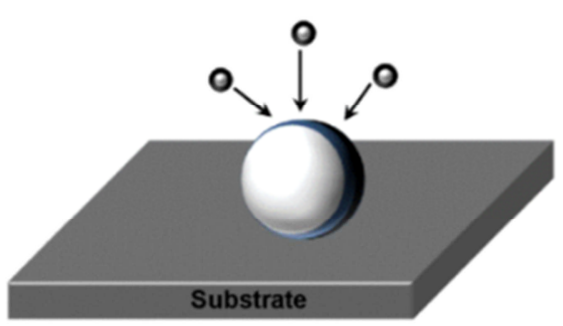

(d) Precipitation at the solid-liquid

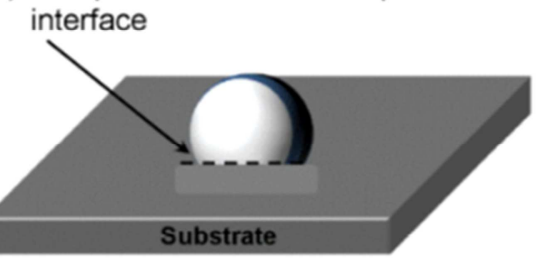

Figure 1. At the edge of the collector-crystal interface where all three phases meet the nucleation probability is high.

\subsection{Conventional Si-NWs Templating Catalysed with Au and $A l$}

Conventional photolithography in conjunction with a $\mathrm{SiO}_{2}$ mask has been shown to be practical for achieving templated NW growth with high yield for noble metals like gold (Au). Figure 2 shows $\mathrm{Si}-\mathrm{NWs}$ growth catalysed with $\mathrm{Au}$, where the position of those NWs is achieved with the photolithography. It has been well established to catalyse $\mathrm{Si}-\mathrm{NWs}$ with $\mathrm{Au}$, for simplicity and a low oxidation rate. However, Au is a deep level trap if we consider those grown NWs for device applications. Semiconductors industries use aluminum (Al) as a dopant to create p-type materials, and it can be used as a catalyst for $\mathrm{Si}-\mathrm{NWs}$ growth. However, $\mathrm{Al}$ is an active element which undergo oxidation very rapidly which supress the NWs growth.

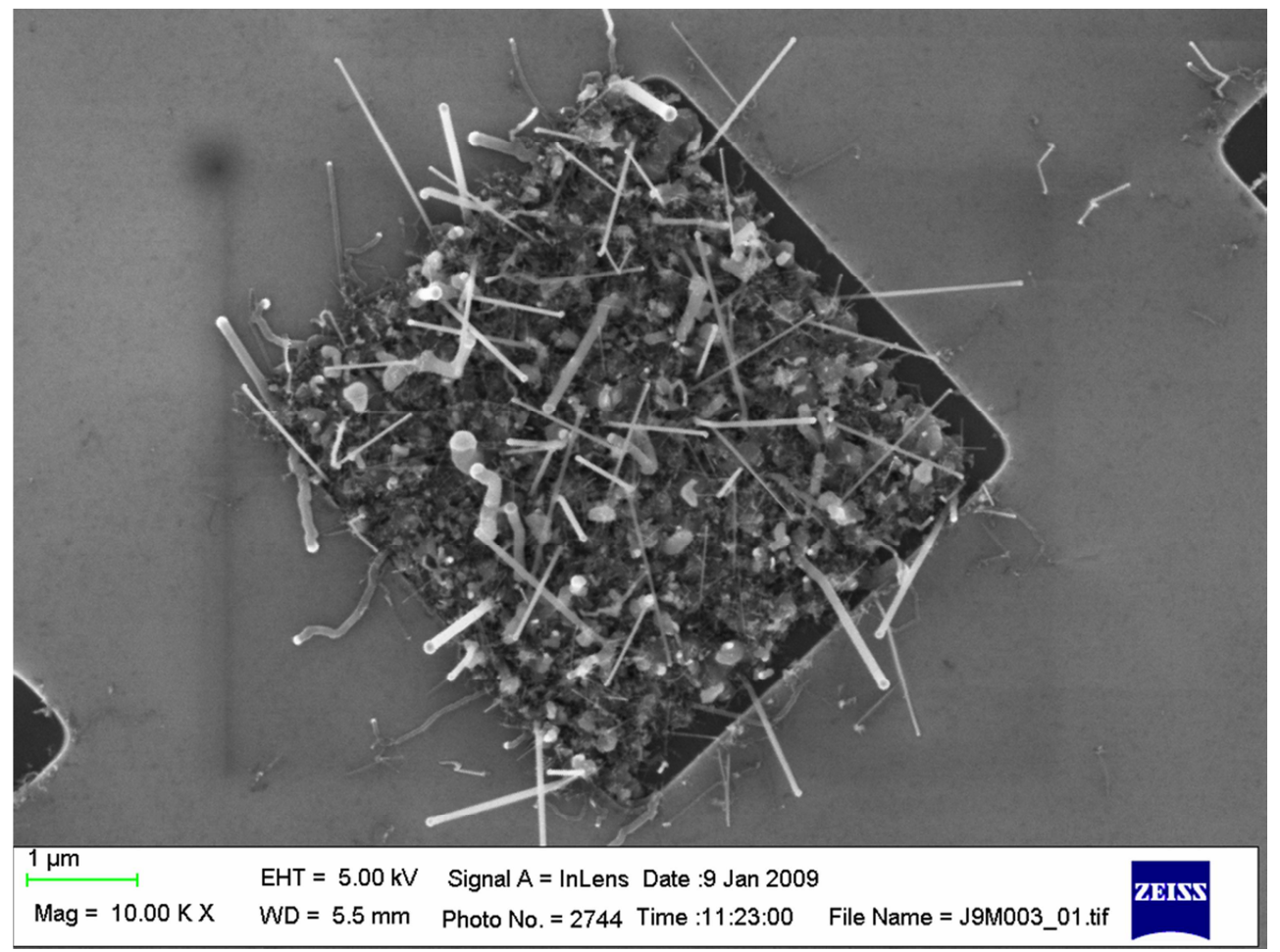

Figure 2. Si-NWs growth catalyzed with 15 nm thick Au on Patterned Si Wafer. 
incorporation of noble metals such as $\mathrm{Au}$ into the $\mathrm{Si}$ [10-12]; furthermore, Al provides lightly p-doped Si NWs [11], without adversely affecting the nanowire growth morphologies, as occurs with doping strategies using only $\mathrm{B}_{2} \mathrm{H}_{6}$.

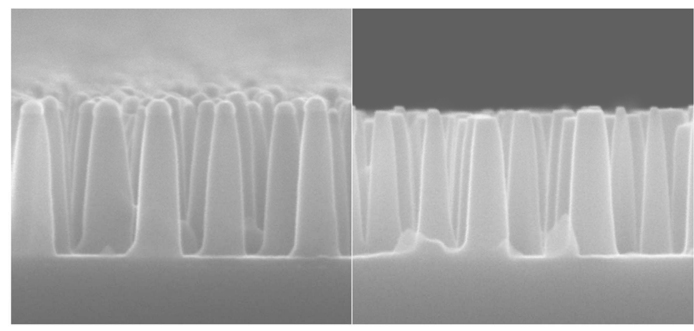

(a)

(b)

Figure 3. Si-NWs catalyzed with Al on Si(111) substrate. (a) Rounded-tops $N W s$, shows at the top of the NWs, Al, (b) Flat-tops NWs, after removing the Al from the top of the NWs using $H F$.

\subsection{Nanoscale Chemical Templating (NCT) Technique}

The novel oxidation scheme of Nanoscale Chemical Templating involves several steps (see figure 4). First, creating an about $50 \mathrm{~nm}$ thick oxide layer on the top of $\mathrm{Si}(111)$ chip, by placing the sample in oxygen environment furnace for certain time according to a calibration chart. Then undertaking a photolithography process via a predesigned mask and developing to get a pre-patterned $\mathrm{SiO}_{2}$. After that in situ $\mathrm{Al}$ deposition in the load lock of the UHVCVD to be transferred to the growth chamber of the UHVCVD for annealing. The Al droplet at the top of $\mathrm{Si}$ in the opening balls-up, while it oxides on the top of $\mathrm{SiO}_{2}$ layer between opening. The last step of $\mathrm{NWs}$ growth is performed as routinely explained [7-11]. While the $6^{\text {th }}$ step depicted in figure 4 describes building $p-n$ junctions for PV application, after removing $\mathrm{Al}$ from the top of the NWs for PV applications. Figure 6 presents details SEM images of areas of several $\mu \mathrm{m}^{2}$, where $\mathrm{SiO}_{2}$ layer appear where there is no growth of $\mathrm{Si}-\mathrm{NWs}$. Nanoscale Chemical Templating process has been optimized reaching growth fidelity of almost $94 \%$ of a single Si-NW/ opening (see figures $6 \& 7$ ).

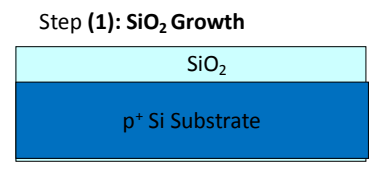

Step (3): In Situ Deposit Al
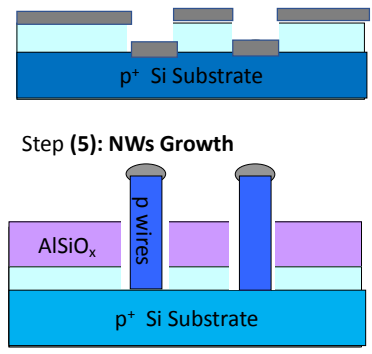

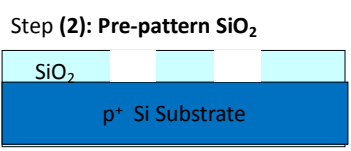

Step (4): In situ Annealing

Step (6): Cap after removing Al

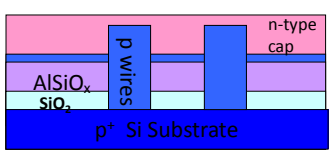

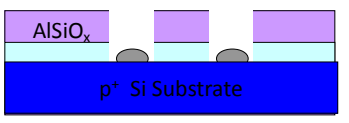

Figure 4. Schematic representations of the various steps involved in the process of Nanscale Chemical Templating (NCT).

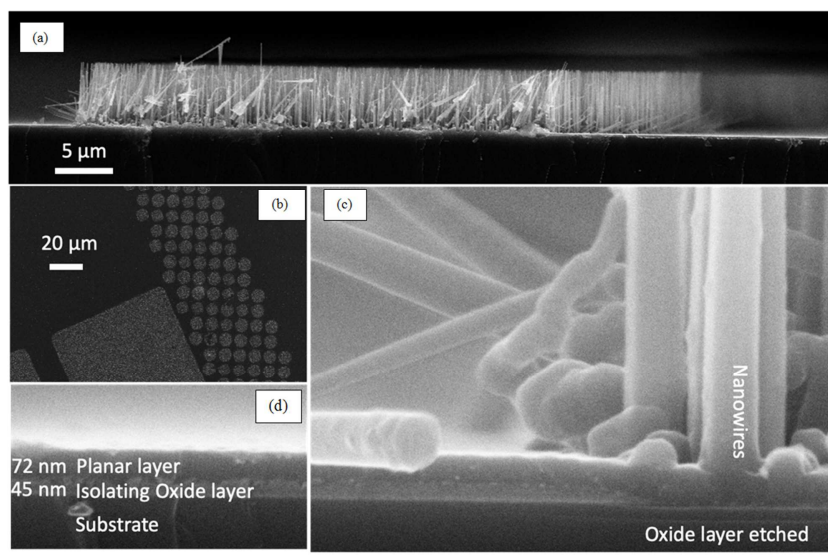

Figure 5. Perpendicular growth of nanowires catalysed with Al, on Si(111) substrate, using Nanoscale Chemical Templating (NCT) technique. (a) SEM micrograph of the side view of NCT growth shows a region where the growth occurs, and regions where there is no growth occurs as $A_{x} O_{x}$ exists (see the thin white layer). (b) SEM image of relatively a low magnification shows bright regions where NWs growth exists and dark regions where there is no $N W$ growth. (c) a high magnification SEM image shows a single NW where the oxide layer was etched and a planner growth on the top of the oxide layer. (d) a SEM image shows the thickness of both the isolating oxide layer and the plannar layer.

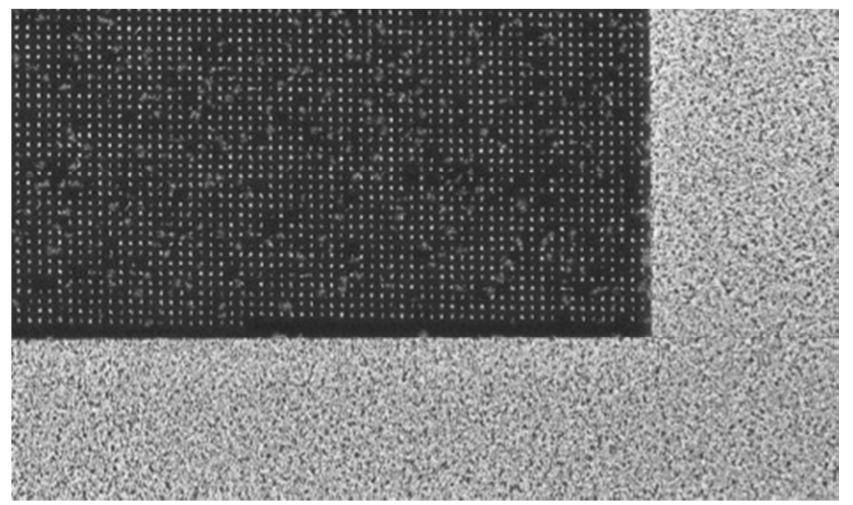

Figure 6. Low magnification SEM image show control growth $\mathrm{Si-NW}$ s using NCT, and regions where Si-NWs growth occur all around the controlled region. (scale bar $10 \mu \mathrm{m}$ ).

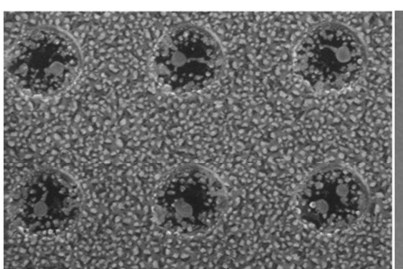

(a)

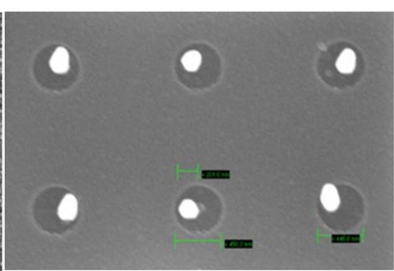

(b)
Figure 7. (a) SEM image shows of $\mathrm{SiO}_{2}$ patterned $\mathrm{Si}(100)$ after annealing. (b) NWs growth perpendicularly of Si(100), where Al at the tops of the NWs, a single NW/ an opening, showing high growth yield at 800 Mtorr growth pressure.

Figures 5, 6 and 7 show SEM micrographs of NCT growth of Si-NWs. The figures display illustrations, cross section and planar SEM images of various stages of NCT, using the typical growth conditions described above. In many applications of patterned NWs, obtaining a single Si NW in each mask opening is critical. Therefore, the growth yield and fidelity of the templating process have been investigated 
as a function of different processing parameters.

\subsection{Nanoscale Chemical Templating (NCT) Using PC or PMMA Microsphere Lithography}

Alternative techniques for lithographic formation of the mask can provide advantages for low-cost processing, especially where a simple repeating pattern is required [1215]. We have evaluated the technique of microsphere lithography to produce regular hexagonal arrays of Al-seeded nanowires. Silica microspheres are commercially available with narrow size distributions, and when dispersed properly on a surface they form a close-packed array that defines gaps through which a material can be deposited. Substrates, $\sim 2$ $\mathrm{cm}^{2}$ cleaved from a p-type, (111), Si wafer, were cleaned using standard techniques which leave a thin oxide on the $\mathrm{Si}$ surface. A drop of $1 \mu \mathrm{m}$ diameter silica microspheres in solution taken directly from the commercially provided stock solution (various suppliers were used) was dispersed onto the substrate. Variable speed spin routines were used on a resist spinner to remove the liquid and disperse the particles [2]. After spinning on the microspheres, the substrates were processed using the sequence described above for the photolithography samples after the resist strip: cleaning with $\mathrm{O}_{2}$ ash, etching with $\mathrm{BOE}$, insertion into the load lock, then deposition of $\mathrm{Al}$ and growth of nanowires. Figures 8 and 9 illustrate the NCT scheme using microspheres. Regions of Al that will act as seeds form at the gaps between microspheres, while on top of the microspheres the Al layer reacts with the oxide and becomes inactive [16].

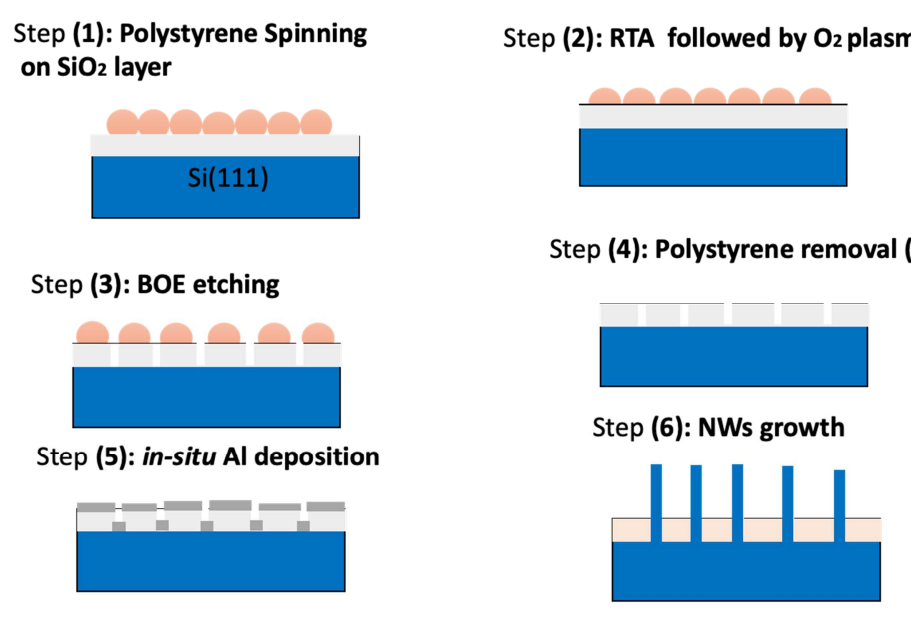

Figure 8. MS Using Polysterene Sphere of $2 \mu$ m Diameter.

\section{Polystyrene MS}

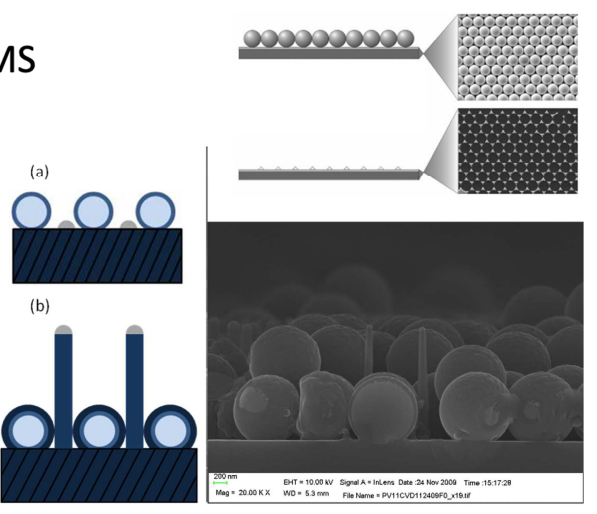

Figure 9. Microsphere of polystyrene and silica processing optimization.

We show the results of this process, where NWs can be seen growing between the gaps of the microsphere array. This process has not been optimized for yield $[10,11,17]$.

\subsection{Si-NWs Growth on Cheap Substrates Such as Al Foil}

1. Nanowires can be grown on Cheap Substrates (Al foil, ...), as shown in figure 10 .

Quick Growth

2. Apply "nanowires" as Light Trapping option for Solar Cells

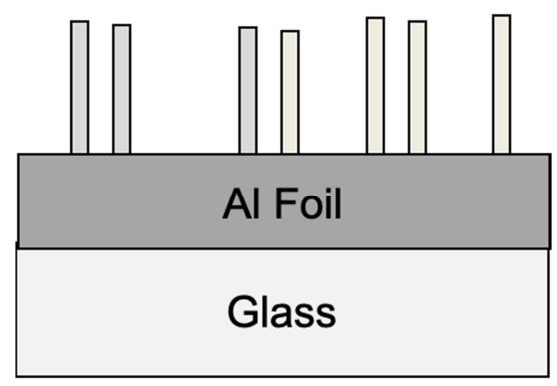

(a)

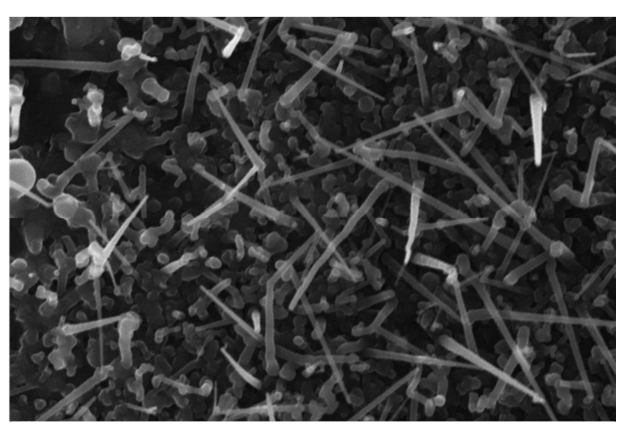

(b)

Figure 10. Nanowires grow on Al foil, at $490^{\circ} \mathrm{C} / 500 \mathrm{mTorr} \mathrm{SiH}_{4} / 30 \mathrm{~min}$. (a) schematic representation, (b) SEM micrograph of Si-NWs growth. 


\subsection{Overview: Applications of Innovative Si-NWs Growth Using UHVCVD}

Nanoscale Chemical Templating (NCT) technique, proposed by Khayyat [5], proposed the concept of controlling the position of the grown NWs catalysed with chemically active elements such as Al. The Ultra High Vacuum Chemical Vapor Deposition UHVCVD reactor usually does not include a metal of generally speaking a catalyst evaporator. So, seed materials have to be evaporated on the substrate in a stand-alone evaporator as routinely practised with gold $\mathrm{Au}$ ) catalyst. However, when we catalyse the nanowires growth with chemically active element such as alluminum (Al) so we have to install it in the tool of UHVCVD.

The proposed concept has been evaluated applying the technique of microsphere lithography to produce regular hexagonal arrays of Al-catalysed Si-NWs. Silica microspheres are commercially available with narrow size distributions, and when dispersed properly using a spinner on the Si substrate surface they form a close-packed array that forms gaps through which a material can be deposited. Si (111) of p-type substrates were cleaned using standard techniques [5-11] which leave a thin oxide on the Si surface. A drop of $1 \mu \mathrm{m}$ diameter silica $\left(\mathrm{SiO}_{2}\right)$ microspheres in solution taken directly from the commercially provided stock solution was dispersed onto the substrate. Then the growth procedure has been adopted as explained earlier [5]. Figure 1 illustrates schematically the various steps of NCT process using microspheres. After spinning in the microspheres (step 1), where it followed by step 2, where then layer of $5 \mathrm{~nm} \mathrm{Al}$ deposited on the surface covering the top surfaces of the microspheres and bare Si surface. Step 3 describes regions of Al that will act as seeds form at the gaps between microspheres, while on top of the microspheres the Al layer reacts with the oxide and becomes inactive $[16,17]$. The final step of this process, step 4, shows the growth of Si-NWs after allowing $\mathrm{SiH} 4$ to flow, providing the required medium of VLS system, to be followed by epitaxial perpendicular SiNWs growth [17, 18, 20].

It has been reported that the overall market of solar photovoltaic installations is growing markedly with a rate exceeding $30 \%$ per annum $[15,19,21]$. The conventional glass PV modules with EVA double sheets at severe weather conditions might results in substantial glass damage and module power reduction. PV modules encapsulate in PC AND/OR PMMA sheets are aiming to overcome these difficulties, in addition to other related issue with the conventional panel. There are several advantages of this adopted technology as follows;

1. Weight: It is approximately weigh 5 times less than the double-glass one, which has also a positive impact on economy.

2. Mechanical resistance: it is virtually unbreakable.

3. Utility: flexible, can be drilled and cut easily.

4. PC reduces the possible damage of the solar cells caused during the placement of the second piece of glass.

5. PC or PMMA modules do not require frames, which are used to protect the glass edges from impact during transport in the current PV modules.

6. PC or PMMA modules have almost no risk of module breakage.

7. Advanced solar cells of Si-NWs can be grown directly on PC substrates (to be studied in details).

8. Most importantly, it can be subsequently transferred to the current manufacturing line of solar panels factory.

9. PC or PMMA with improved properties can be provided from companies such as SABIC.

10. Using PMMA to fabricate Si-NWs PV cells is an innovative economical solution (to be studied in details).

\section{Conclusions}

The Ultra High Vacumm Chemical Vapor Deposition UHCVD reactor, which was used in the previous studies, does not include an aluminum evaporator, so we have to install it in the tool separately. The study includes several growth approaches of Si-NWs, where main conclusive remarks can be stated as follows;

1- The structure and electronic characteristics of these Alcatalyzed silicon nanowires ( $\mathrm{Si}-\mathrm{NWs}$ ) are highly relevant to many applications, for example, in photovoltaics.

2- Nanoscale Chemical Templating (NCT) method has been employed to achieve controlled spatial placement of SiNWs has using $\mathrm{SiO}_{2}$. PC or PMMA nanospheres can be used similarly, as a mask and $\mathrm{Al}$ as the seed material.

4- The NCT with PC microspheres method is economic, as it requires fewer steps compared to conventional patterning approaches, not requiring lift off of a metal layer or removal of the mask.

5- The methodology used in achieving controlled placement of single NWs seeded with $\mathrm{Al}$ is an example of proving the concept of NCT technique, more oxygen-reactive materials such as $\mathrm{Sn}, \mathrm{Sb}, \mathrm{In}, \mathrm{Ga}$, and Ti templated by NCT will have more advanced applications in nanodevices fabrications.

\section{Funding Information}

This research has been done as part of the employment at KACST.

\section{Conflict of Interest}

The author declares that there is no conflict of interest regarding the publication of this paper.

\section{Acknowledgements}

The author would like to thank Dr T. C. Chen, T. J. Watson Research Center for his scientific insights throughout working on this project. 


\section{References}

[1] B. J. Huang, T. H. Lin, W. C. Hung and F. S. Sun, Performance evaluation of solar photovoltaic/thermal systems, Solar Energy Volume 70, Issue 5, 2001, Pages 443-448.

[2] Li Gong, Jianguo $\mathrm{Lu}$ and Zhizhen Ye, Transparent and conductive Ga-doped $\mathrm{ZnO}$ films grown by $\mathrm{RF}$ magnetron sputtering on polycarbonate substrates, Solar Energy Materials and Solar Cells Volume 94, Issue 6, June 2010, Pages 937941.

[3] C. J. Brabec, N. S. Sariciftci \& J. C. Hummelen, Plastic solar cells. Advanced Functional Materials, 11 (1), 2001, 15-26.

[4] W. R. Zeng, S. F. Li, W. K. Chow, Preliminary Studies on Burning Behavior of Polymethylmethacrylate (PMMA), Journal of Fire Sciences. 20 (4), 2002, 297-317.

[5] B. A. Wacaser, M. C. Reuter, M. M. Khayyat, C. Y. Wen, R. Haight, S. Guha, F. M. Ross, Growth Systems, Structure, and Doping of Aluminum Seeded Epitaxial Silicon Nanowires, Nano letters, Vol 9, 2009.

[6] M. M. Khayyat, B. Aissa, E. H. Abdul-Hafidh, M. Nedil, Controlled-Crystal-Growth and Structures of Silicon Nanowires for Smart Applications, SDRP Journal of Nanotechnology \& Material Science, 2015.

[7] M. Khayyat, B. Wacaser, M. Reuter, F. Ross, D. Sadana and Tze Chin, Nanoscale chemical templating of Si-NWs seeded with Al, Nanotechnology 24, 2013, 235301.

[8] M. Khayyat, B. Wacaser, D. Sadana, Nanoscale chemical templating with oxygen reactive materials, USPTO Patent number 8349715 .

[9] H. J. Fan, P. Werner and M. Zacharias, Semiconductor nanowires: from self-organization to patterned growth, Small 2, 2006, 700-17.

[10] B. A. Wacaser, M. M. Khayyat, M. C. Reuter, D. K. Sadana, F. M. Ross, Technical advantages and challenges for coreshell micro/ nanowire large area PV devices, Photovoltaic Specialists Conference (PVSC), 35th IEEE, 2010.
[11] B. A. Wacaser, M. C. Reuter, M. M. Khayyat, R. Haight, S. Guha and F. M. Ross, The Role of Microanalysis in Micro/Nanowire-Based Future Generation Photovoltaic Devices, Microscopy and Microanalysis, Volume 16, Supplement S2, 2010.

[12] M. M. Khayyat, B. A. Wacaser, M. C. Reuter, D. K Sadana, Templating silicon nanowires seeded with oxygen reactive materials, Saudi International Electronics, Communications and Photonics Conference (SIECPC), 2011.

[13] Y. Huang and C. M. Lieber, Integratednanoscale electronics and optoelectronics: exploring nanoscale science and technology through semiconductor nanowires Pure Appl. Chem. 76, 2004 2051-68.

[14] B. M. Kayes, H. A. Atwater and N. S. Lewis, Comparisonof the device physics principles of planar and radial $\mathrm{p}-\mathrm{n}$ junction nanorod solar cells J. Appl. Phys. 97, 2005, 114302.

[15] M. Law, J. Goldberger and P. D. Yang, Semiconductor nanowires and nanotubes Annu. Rev. Mater. Res. 34, 2004, 83-122.

[16] L. Samuelson et al, Semiconductor nanowires for 0D and 1D physics and applications, Physica E 25, 2004, 313-8.

[17] E. I. Givargizov, Fundamental VLS growth J. Cryst. Growth $31,1975,20-30$.

[18] B. A. Wacaser, K. A. Dick, J. Johansson, M. T. Borgstrom, K. Deppert and L. Samuelson, Preferential interface nucleation: an expansion of the VLS growth mechanism for nanowires Adv. Mater. 21, 2009, 153-65.

[19] R. S. Wagner and W. C. Ellis, Vapor-liquid-solid mechanism of single crystal growth, Appl. Phys. Lett. 4, 1964, 89-90.

[20] B. M. Kayes, M. A. Filler, M. C. Putnam, M. D. Kelzenberg, N. S. Lewis and H. A. Atwater, Growth of vertically aligned $\mathrm{Si}$ wire arrays over large areas $(>1 \mathrm{~cm} 2)$ with $\mathrm{Au}$ and $\mathrm{Cu}$ catalysts Appl. Phys. Lett. 91, 2007, 103110.

[21] J. Westwater, D. Gosain, S. Pand, Control of the size and position of silicon nanowires grown via the vapor-liquid-solid technique, Japan. J. Appl. Phys., 1 36, 1997, 6204-9. 